\title{
Study on the Relationship between Nantong Blue Calico and Paper-Cut Art
}

\author{
Hong-Guang $\mathrm{YE}^{1, \mathrm{a}}$, Bing-Ying OU ${ }^{1, \mathrm{~b}}$, Qiang $\mathrm{LI}^{2, \mathrm{c}}$ and Bin $\mathrm{LI}^{1, \mathrm{~d}^{*}}$ \\ ${ }^{1}$ College of Fashion, Wuhan Textile University, Wuhan, 430073, China \\ ${ }^{2}$ Editorial Department of Fashion Guide, Wuhan Textile University, Wuhan, 430073, China \\ a836001463@qq.com, b995901055@qq.com, 'liqiang800121@foxmail.com, ${ }^{d}$ libin790121@qq.com
}

Keywords: Blue calico, paper-cut, relationship.

\begin{abstract}
There are a lot of similarities between Nantong blue calico and kirigami. There is a systematic study on the relationship between the Nantong blue calico and the art of paper cutting by using the method of historical documents and comparative study. Research results show that, firstly, the stencil design of Nantong blueprint cloth inherits and develops the performance of paper-cut patterns. Secondly, Nantong blue calico and paper-cut patterns have similarities in cultural heritage, namely Nantong blue calico and paper-cut patterns are using harmonics, symbolic form of artistic expression.
\end{abstract}

\section{Introduction}

Nantong blue calico and paper-cut art are closely related, mainly in stencil design and production. From emergence time of the stencil design of Nantong blue calico and the art of paper cutting to see, the art of paper cutting is far earlier than the emergence of the stencil of Nantong blue calico. And the blue calico and paper-cut are deeply rooted in the folk. It is obvious that artistic expression of paper cutting is borrowed by Nantong blue calico. From the stencil pattern of Nantong blue printed cloth and the paper-cut pattern to see, on the one hand, the stencil design of Nantong blue printed cloth inherited the technique of expression of the paper-cut patterns, on the other hand, as Nantong unique art of printing and dyeing, Nantong blue calico is different from artistic expression of the paper-cut, but both the pattern has interlinked in the cultural heritage.

\section{Discussions and Analyses}

Nantong Blue Calico and Paper-Cut Patterns have relevance to Technique of Expression

The paper-cut is with scissors or a knife as a tool for the creation of hollow art, the carrier of paper cutting includes not only the paper, but also includes gold and silver foil, bark, leaves, cloth, leather and other sheet materials. In a broad sense, the stencil design of Nantong blue print cloth is a variant of the art of paper cutting. Therefore, the paper-cut art pattern and Nantong blue calico patterns have a certain relationship.

(1)The Stencil Design of Nantong Blue Print Cloth Inherited Technique of Expression of Paper-Cut Patterns

As is known to all, the true sense of the art of paper cutting appeared in the northern and Southern Dynasties (AD420-AD589), a scrap of paper-cut with a pattern featuring twin horses and flower mission on The Northern Dynasties (AD386-AD581) unearthed in Xinjiang Turpan Gaochang ruins in 1959.[1] This shows that the appearance of paper-cut is at least as early as six hundred years than Nantong blue calico's predecessor -- "Yaoban cloth". The stencil design of Nantong blue calico and paper-cut patterns have similarities in expression technique, performance method of paper cutting patterns contains expression technique of the stencil design of Nantong blue calico. Nantong blue calico pattern is divided into decorative white patterns on blue cloth and decorative blue patterns on white cloth, therefore, its stencil is divided into the stencil of blue cloth (Figure 1) and the stencil of white cloth (Figure 2). Two kinds of the stencil are similar to yin carving and yang carving in the 
paper-cut. Paper-cut of Yin carving is carved to the original contour line, to retain the part outside of contour lines. So the characteristics of the Yin carved paper-cut are that its lines are not necessarily interconnected, and the overall works are blocked. Paper-cut of the yang carved is completely opposite to the Yin carved. The feature is to retain the contour line of the original, cut out the blank space outside the contour line. Its every line is connected to each other. In fact, the patterns on the stencil of white cloth of Nantong blue calico usually use the technique of expression of the yang carved, and part of the border uses the technique of expression of the yin carved. In the stencil of white cloth of "Dragon and Phoenix mean auspicious"(Figure 3), Phoenix and peony patterns are carved by the technique of expression of the yang carved. Theme pattern presents the form of blue flower in white cloth through the scraping and dipping. But plant pattern on the border is used in the way of Yin carved, so the pattern at the border section shows the form of white flowers in blue cloth. Similarly, there are the same methods in the paper-cut patterns. It is obvious that the paper-cut patterns are more likely to be realized than the stencil design of Nantong blue calico in the performance practices. Therefore, the author believes that the stencil design of Nantong blue calico must be the impact of paper cutting patterns.

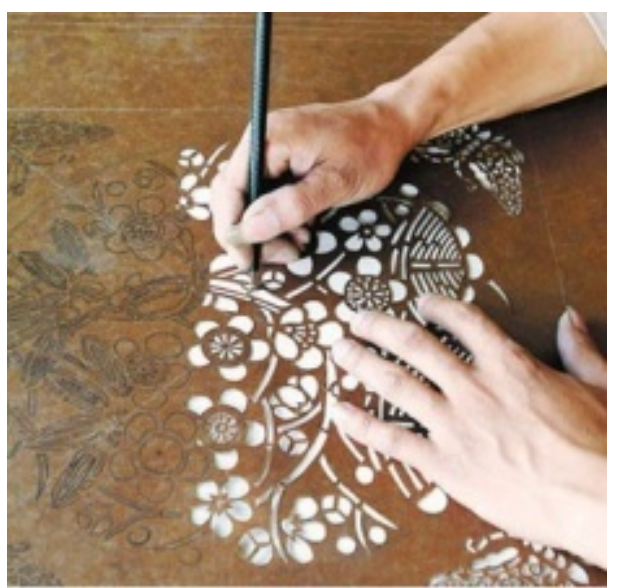

Fig. 1 the stencil of blue cloth

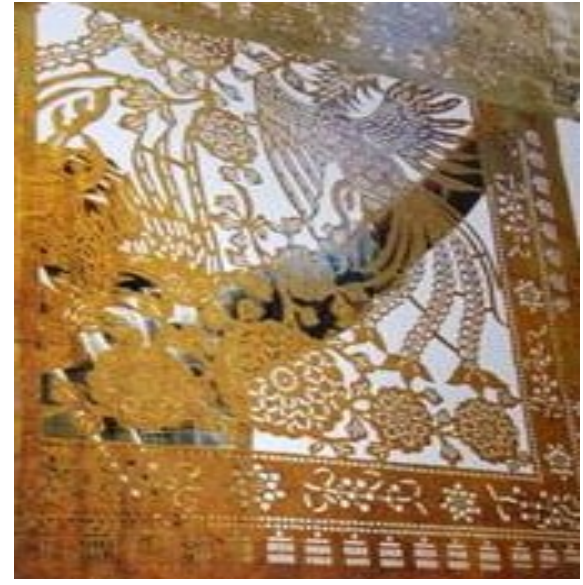

Fig. 2 the stencil of white cloth

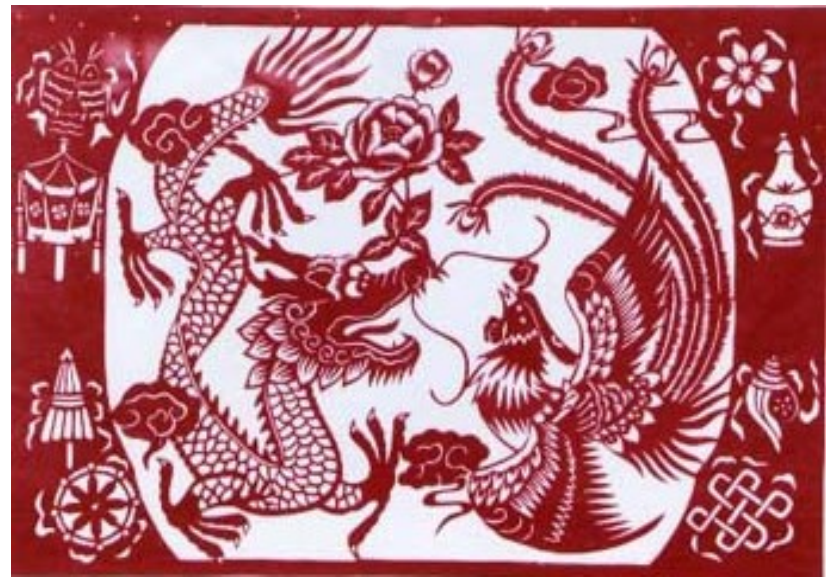

Fig. 3 paper-cut of Dragon and Phoenix mean auspicious 


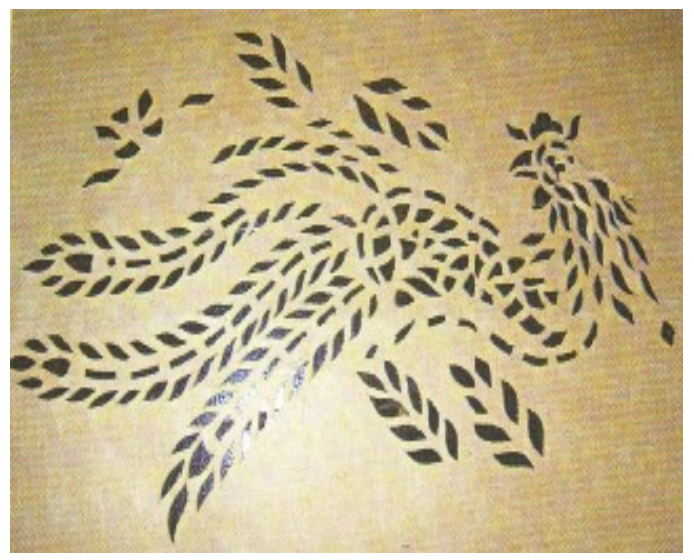

Fig. 4 A technique by using a small knife to carve out an intermittent pattern

\section{2) The Production of the Stencil of Nantong Blue Calico Makes Improvement to Expression of Paper-Cut Patterns}

We can find that the stencil of Nantong blue calico makes improvement and innovation to skills of paper-cut through carving skills of the stencil. There is a term used in the carving skills of the stencil of Nantong blue calico, which is called "using a small knife to carve out an intermittent pattern". It refers to something that the length of the line is divided into several sections, each section with "bridge" connected, "using a small knife to carve out an intermittent pattern" the length is not longer than $2-3 \mathrm{~cm}$, the connection is not less than $0.2 \mathrm{~cm}$, without prejudice to the pattern for the principle, to achieve " the lines are broken, but the meaning of expression is complete"(Figure 4).[2] There are two reasons for the generation of the "using a small knife to carve out an intermittent pattern" technique: one is to prevent the stencil damage in the process of scraping due to too long or too fine lines; the two is to prevent leakage scraping impact the integrity of the stencil in the process of scraping. So, Nantong blue calico line and surface must be broken off, the shorter the better, otherwise it is not suitable for printing and dyeing. It is not difficult to understand that the traditional Nantong blue calico is widely used for continuous dot and expression technique of "using a small knife to carve out an intermittent pattern". And the line and the face of paper cuts must be connected, the line is connected the more tightly the better. On the contrary, the paper-cut cannot be completed. The author believes that the production of the stencil of Nantong blue calico makes reasonable improvement and innovation to expression of paper-cut patterns under the principle of suitability for printing and dyeing.

\section{Nantong Blue Calico and Paper-Cut Patterns have Similarities in Cultural Heritage}

Nantong blue calico and paper-cut belong to daily necessities in the farming community, they must have the similarities in the pattern, which reflects the people's aesthetic habits and ways of thinking at that time.

First of all, Nantong blue calico and paper-cut patterns use homophonic art form to reflect the happy and peaceful atmosphere. These pattern which "Magpie in the branch of plum tree"(Figure 5) "Auspicious omen”(Figure 6) "Be promoted" "Become the champion" "Happiness comes" are used homophonic ways. In Chinese culture, "Magpie in the branch of plum tree” means "Have good news", reflects the festive atmosphere. The pronunciation of " $\mathrm{Ji}$ " in Auspicious omen and "Auspicious" is the same; The pronunciation of "Qing" and "festivity" is the same; The pronunciation of "Yu" and "surplus" is the same; The combination of these patterns is meaning of "Auspicious Omen". "Three halberd into the bottle" and "Be promoted" are partial tone. "auspicious" and "Halberd”, "land” and "promote", "bat" and "happiness" are used to reflect the meaning of patterns by the way of partial tone.

Secondly, Nantong blue calico and paper-cut patterns use the method of symbolic expression. For example: peony is a symbol of riches and honour; pomegranate is a symbol of fertility; plum 
blossoms, orchid, bamboo and chrysanthemum are the symbol of man's integrity; pine and crane are the symbol of longevity; dragon and phoenix are the symbol of nobility; the lion is a symbol of safeness and so on. Phoenix and peony in paper-cut pattern of Phoenix and Peony(Figure 7) are a symbol of riches and honour. Pattern in the blue calico of "Kylin delivers the child"(Figure 8) reflects the people to pray for members of descendants. Kylin is benevolent beast in the ancient Chinese legend, and it is a symbol of good luck and can bring children to people. There is the pattern that the boy holds lotus flower in "Kylin delivers the child". Lotus flower will produce Semen nelumbinis. Semen nelumbinis is a symbol of many descendants.

Finally, methods of homophonic and symbolism are used together in Nantong blue calico and paper-cut patterns. For example: in Chinese culture, in paper-cutting pattern of "Happiness and longevity come"(Figure 9), the pronunciation of "bat" and "happiness" is the same, "money" and "front" are partial tone, "Hole" compare to "eye", and the middle of the pattern is a symbol of longevity. The combination of these patterns is meaning of "Happiness and longevity come".

Similarly, Nantong blue printed cloth also has such an interpretation, "Happiness comes"(Figure 10) which is enshrined in the Nantong blue calico museum is very well reflected in the method of artistic expression homophonic and symbol. In fact, this blue calico contains four groups of theme patterns which are "Happiness comes", "Phoenix and peony" and "safeness and prosperity", "Three plants grown in winter". "Happiness comes" and "safeness and prosperity" use the artistic expression of the partial tone, "Phoenix and peony" and "Three plants grown in winter" use the artistic expression of symbolic. The patterns use the symmetrical composition which makes the four groups of patterns cleverly combination together, it expressed the people yearning for life rich and safeness and noble personality.

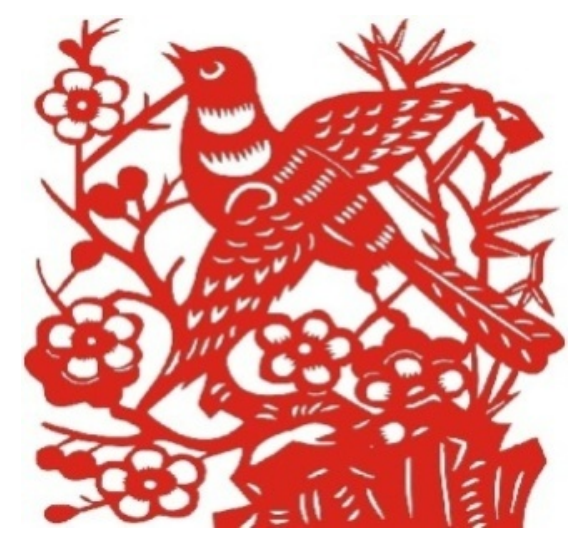

Fig. 5 paper-cut of Have good news

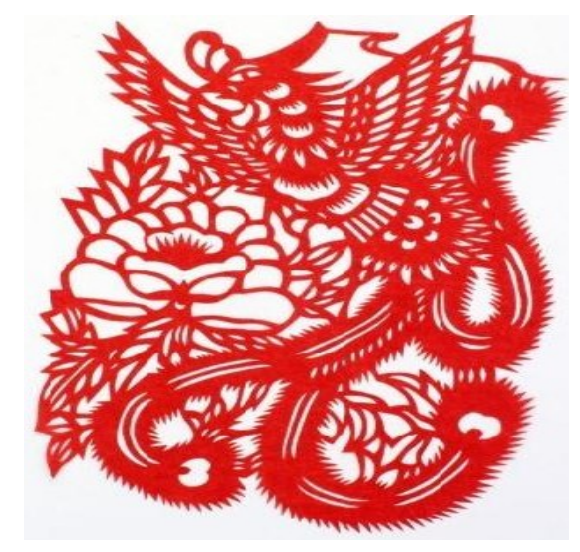

Fig. 7 paper-cut of Phoenix and Peony

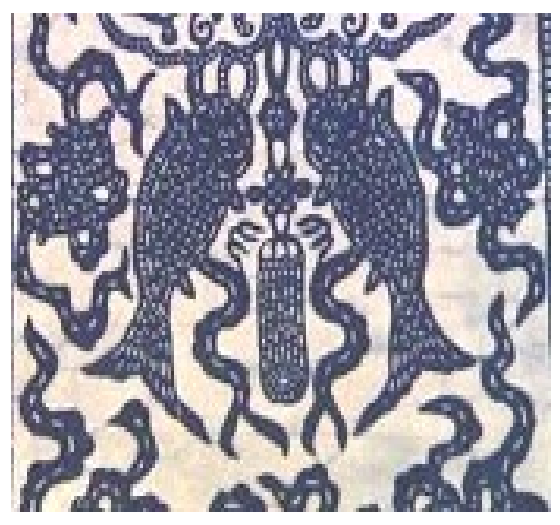

Fig. 6 the blue calico of Auspicious omen

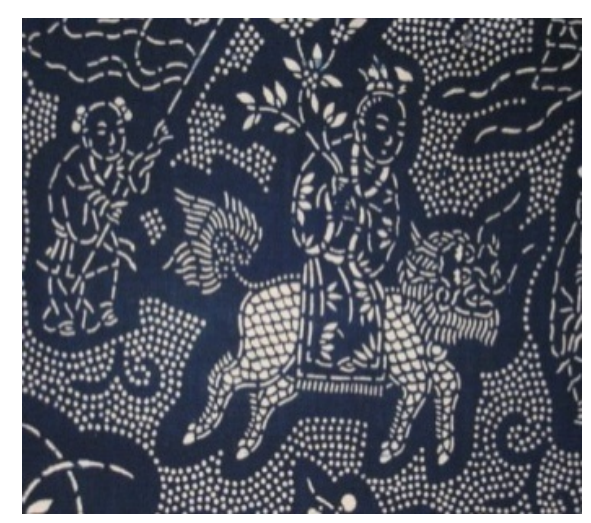

Fig. 8 the blue calico of Kylin delivers the child 


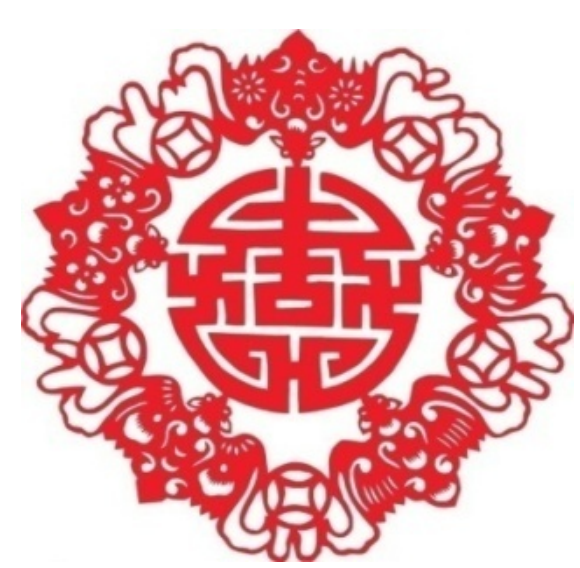

Fig. 9 paper-cut of Happiness and longevity come

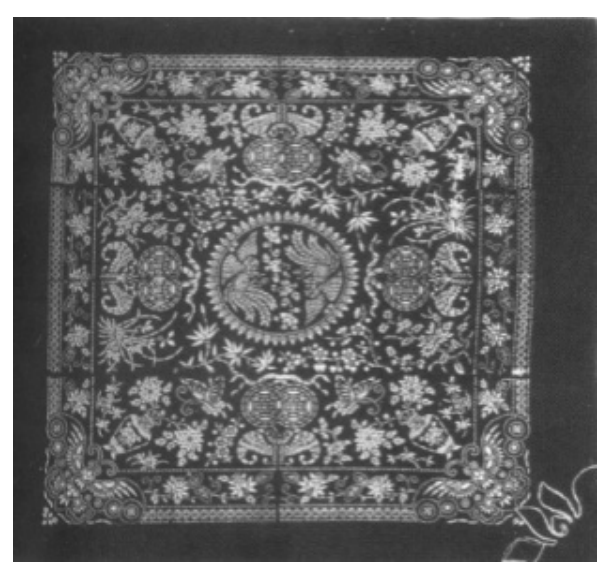

Fig. 10 the blue calico of Happiness comes

\section{Conclusion}

Nantong blue calico patterns and paper-cut patterns has great similarities in the composition and form of expression. It shows that the two folks traditional crafts have similarities in culture, mapping out culture of blessing, reproduction, plain and romantic. Because the paper-cut appearance is much earlier than Nantong blue calico, meanwhile, the realization of the patterns of paper cutting is easier and more convenient than the production of the stencil design of Nantong blue printed cloth. So there is every reason to believe that the art of paper cutting has greatly promoted the development of the stencil of Nantong blue calico.

\section{References}

[1] Zhou Yuebing: A Preliminary Study of Chinese Folk Art -- The Origin And Characteristic of The Art of Paper Cutting (Popular Literature And Art, China 2011).

[2] Wu Yuanxin, Wu Lingshu: The Spirit of Scraping And Printing -- Chinese Blue Calico (Harbin: Heilongjiang People's Publishing Press, China 2011). 